\title{
Th1 cytokines, programmed cell death, and alloreactive $T$ cell clone size in transplant tolerance
}

\author{
Koji Kishimoto, ${ }^{1}$ Sigrid Sandner, ${ }^{1,2}$ Jaime Imitola, ${ }^{1,2}$ Masayuki Sho, ${ }^{1,2}$ Yongsheng $\mathrm{Li},{ }^{3}$ \\ Peter B. Langmuir, ${ }^{4}$ David M. Rothstein, ${ }^{5}$ Terry B. Strom, ${ }^{3}$ Laurence A. Turka, ${ }^{6}$ \\ and Mohamed H. Sayegh ${ }^{1,2}$
}

1Laboratory of Immunogenetics and Transplantation, Brigham and Women's Hospital,
${ }^{2}$ Division of Nephrology, Children's Hospital, and
${ }^{3}$ Division of Immunology, Beth Israel Deaconess Medical Center, Harvard Medical School, Boston, Massachusetts, USA
${ }^{4}$ Children's Hospital of Philadelphia, Philadelphia, Pennsylvania, USA
${ }^{5}$ Department of Internal Medicine, Yale Medical School, New Haven, Connecticut, USA
${ }^{6}$ Department of Medicine, University of Pennsylvania, Philadelphia, Pennsylvania, USA

Address correspondence to: Mohamed H. Sayegh, Laboratory of Immunogenetics and Transplantation,

Brigham and Women's Hospital, Harvard Medical School, 75 Francis Street, Boston, Massachusetts 02115, USA.

Phone: (617) 732-5239; Fax: (617) 732-5254; E-mail: msayegh@rics.bwh.harvard.edu.

Terry B. Strom, Laurence A. Turka, and Mohamed H. Sayegh contributed equally to this work.

Received for publication January 2, 2002, and accepted in revised form April 30, 2002.

\begin{abstract}
The Th1 cytokines IL- 2 and IFN- $\gamma$, which inhibit $\mathrm{T}$ cell proliferation and promote activation induced cell death, may be required to diminish alloreactive $\mathrm{T}$ cell numbers and to foster tolerance across full allogeneic barriers. However, we hypothesized that these cytokines might be dispensable when the alloreactive $\mathrm{T}$ cell clone size is relatively small, as is seen in recipients of minor-mismatched grafts. We show that alloreactive $\mathrm{T}$ cell clone size of $\mathrm{C} 57 \mathrm{BL} / 6$ mice against multiple minor-mismatched $129 \mathrm{X} 1 / \mathrm{sv}$ mice was $\sim 4-9$-fold smaller than that against MHC-mismatched BALB/c mice. In the MHCmismatched combination, CD28-B7 blockade by CTLA4Ig induced long-term graft survival in wildtype recipients, but this treatment was ineffective in $I F N \gamma^{-}$or $I L-2^{-/-}$recipients. In contrast, in the minor-mismatched combination, CTLA4Ig induced long-term allograft survival in wild-type, IFN $\gamma^{\prime-}$, and $I L-2^{-/-}$recipients. Bcl- $\mathrm{x}_{\mathrm{L}}$ transgenic animals, which are defective in "passive" T cell death, are likewise sensitive to the effects of CTLA4Ig only in the setting of the minor-mismatch grafts. Therefore, the alloreactive $\mathrm{T}$ cell clone size is an important determinant affecting the need for $\mathrm{Th} 1$ cytokines and $T$ cell death in tolerance induction. These data have implications for the design of tolerance strategies in transplant recipients with varying degrees of MHC mismatching.
\end{abstract}

J. Clin. Invest. 109:1471-1479 (2002). doi:10.1172/JCI200214947.

\section{Introduction}

Recent studies indicate that both IFN- $\gamma$ and IL-2 play a dual role in the immune system. On the one hand, these cytokines have redundant roles (IL-2 as a growth factor and IFN- $\gamma$ as a proinflammatory cytokine), which may be replaced by other cytokines. On the other hand, these cytokines inhibit T cell expansion (IFN- $\gamma$ ) and promote apoptosis (IL-2 and IFN- $\gamma$ ) of antigenactivated $T$ cells that serve to limit immune responses (1-6). This negative feedback loop helps to maintain $\mathrm{T}$ cell homeostasis and restrains autoimmunity (7).

Recent studies by our group and others have examined the role of apoptosis in transplantation tolerance. It has been shown previously that IFN- $\gamma$ limits alloreactive $\mathrm{T}$ cell proliferation (8) and promotes apoptosis of antigen-specific $\mathrm{CD}^{+}$and $\mathrm{CD} 4^{+} \mathrm{T}$ cells $(4,6)$. Similarly, IL-2 is required to prime alloreactive T cells for activation-induced cell death (AICD) $(1,9)$ with consequent peripheral deletion of donor-reactive $\mathrm{T}$ cells in recipients of MHC-mismatched allografts (10-12). Although blockade of CD28-B7 and/or CD154-CD40 T cell costimulatory pathways results in long-term allograft acceptance in several transplant models (reviewed in refs. 13, 14), recent studies have demonstrated that these strategies cannot induce long-term allograft survival in MHC-mismatched donor-recipient combinations when the recipients lack either IFN- $\gamma$ or IL-2 (10, 11,15-17). Complementary data showing that animals expressing $\mathrm{Bcl}-\mathrm{x}_{\mathrm{L}}$ as a transgene in the $\mathrm{T}$ cell lineage also resist transplant tolerance induction across MHC barriers using costimulatory blockade (11) further support the role of $\mathrm{T}$ cell death in transplantation tolerance.

The primacy of $\mathrm{T}$ cell apoptosis, as opposed to regulation, in the transplant studies cited above stands in contrast to the data in many autoimmune models, where, for example, immune deviation by itself is sufficient to prevent or reverse the pathologic $T$ cell response $(18,19)$. We have hypothesized that one reason for this distinction might be the difference in the size of the pool of responding $\mathrm{T}$ cells, which is large in the case of MHC-mismatched transplants and presumably smaller in the case of autoimmune disease models. Supporting this concept, we have found that equally robust immune deviation with an anti-IL-12 
$\mathrm{mAb}$ was able to induce tolerance in recipients of minor-mismatched allografts, but was not successful in MHC-mismatched grafts (20).

Our "T cell clone size" hypothesis predicts that mechanisms that limit $\mathrm{T}$ cell expansion and/or promote $\mathrm{T}$ cell apoptosis leading to significant reduction of alloreactive $T$ cells need not be required for tolerance induction across minor histocompatibility barriers, where it is presumed that the responding alloreactive $\mathrm{T}$ cell clone size is much smaller than that across MHC barriers. Here, we describe studies in a series of complementary models designed to test this hypothesis.

\section{Methods}

Mice. C57BL/6 (H-2 $\left.2^{\mathrm{b}}\right), 129 \mathrm{X} 1 / \mathrm{sv}(129)\left(\mathrm{H}-2^{\mathrm{b}}\right), \mathrm{C} 3 \mathrm{H} / \mathrm{He}$ $\left(\mathrm{H}-2^{\mathrm{k}}\right), I F N-\gamma^{\prime-} \mathrm{C} 57 \mathrm{BL} / 6\left(\mathrm{H}-2^{\mathrm{b}}\right), I L-2^{-/-} \mathrm{C} 57 \mathrm{BL} / 6\left(\mathrm{H}-2^{\mathrm{b}}\right)$, C57BL/6J-Igh ${ }^{\mathrm{a}}$-Thy1 ${ }^{\mathrm{a}}$ (B6-Thy1.1) $\left(\mathrm{H}-2^{\mathrm{b}}\right), \mathrm{C} 57 \mathrm{BL} / 6 \times$ $\mathrm{BALB} / \mathrm{c}$ F1 (CB6F1) $\left(\mathrm{H}-2^{\mathrm{b} / \mathrm{d}}\right)$, and C57BL/6 × 129P3 $(\mathrm{B} 6129 \mathrm{~F} 1)\left(\mathrm{H}-2^{\mathrm{b}}\right)$ mice aged 6-8 weeks were purchased from The Jackson Laboratory (Bar Harbor, Maine, USA), and $\mathrm{BALB} / \mathrm{c}\left(\mathrm{H}-2^{\mathrm{d}}\right)$ mice aged $6-8$ weeks were purchased from Taconic Farms (Germantown, New York, USA). The Bcl- $x_{\mathrm{L}}$ transgenic animals were a kind gift of Gabriel Nunez (University of Michigan, Ann Arbor, Michigan, USA) and were inbred in our animal facility (11).

Enzyme-linked immunosorbent spot analysis. Enzymelinked immunosorbent spot (ELISPOT) plates (Polyfiltronics Inc., Rockland, Massachusetts, USA) were coated with $4 \mu \mathrm{g} / \mathrm{ml}$ of rat anti-mouse IFN- $\gamma$ (R4-6A2) capturing mAb's in sterile PBS overnight. The plates were then blocked for 1.5 hours with sterile PBS containing $1 \%$ BSA and washed with sterile PBS. Splenocytes $\left(1.2 \times 10^{6}\right.$ in $0.2 \mathrm{ml}$ AIM-V medium $)$ were then placed in each well in the presence of $1.2 \times 10^{6}$ irradiated $(20 \mathrm{~Gy})$ syngeneic or allogeneic splenocytes (as antigen-presenting cells) and cultured for 48 hours at $37^{\circ} \mathrm{C}$ in $5 \% \mathrm{CO}_{2}$. For detection of spots, $5 \mu \mathrm{g} / \mathrm{ml}$ of biotinylated rat antimouse IFN- $\gamma \mathrm{mAb}$ (XMG 1.2) was used, followed by 2 hours of incubation with streptavidin $\mathrm{D}$ horseradish peroxidase (Vector Laboratories, Burlingame, California, USA) diluted at 1:2,000 in PBS $0.025 \%$ Tween. All mAbs were purchased from PharMingen (San Diego, California, USA). After washing, the plates were developed using $0.8 \mathrm{ml}$ of 3-amino-9-ethylcarbazole (Pierce Chemical Co., Rockford, Illinois, USA; $10 \mathrm{mg}$ dissolved in $1 \mathrm{ml}$ dimethyl formamide) mixed with $24 \mathrm{ml}$ of $0.1 \mathrm{M}$ sodium acetate, pH 5.0, containing $12 \mu \mathrm{H}_{2} \mathrm{O}_{2}$. The resulting spots were counted on a computer-assisted ELISPOT image analyzer (T Spot Image Analyzer; Cellular Technology Ltd., Cleveland, Ohio, USA) $(21,22)$.

Flow-cytometric analysis of $T$ cell proliferation. Spleen and lymph nodes from donor B6-Thy1.1 mice were harvested and single-cell suspensions were labeled with 6-carboxyfluorescein diacetate succinimidyl ester (CFSE) (Molecular Probes, Eugene, Oregon, USA), as previously described $(23,24)$. Labeled donor cells $\left(30 \times 10^{6}\right)$ were injected intravenously into nonirradiated $\mathrm{CB} 6 \mathrm{~F} 1$ or B6129F1 mice (all Thy1.2+), and spleen and lymph nodes were harvested from recipient mice 72 hours after donor cell injection. Single-cell suspensions were analyzed by flow cytometry after labeling with phycoerythrin-conjugated anti-Thy1.1 and either peridinin chlorophyll protein-conjugated anti-CD4 or anti-CD8 $\mathrm{mAb}$ (PharMingen). Unlabeled $\mathrm{Ab}$ against CD16/CD32 was used to block $\mathrm{Fc}$ receptor binding. The cells were analyzed on a FACScalibur (Becton-Dickinson Immunocytometry Systems, San Jose, California, USA), and data were analyzed using FlowJo software (Tree Star Software, San Carlos, California, USA).

Fusion proteins and $m A b$ 's. The anti-CD154 hybridoma MR1 was a kind gift from Randy Noelle (Darmouth Medical School, Hanover, New Hampshire, USA), and the $\mathrm{Ab}$ was manufactured by Bioexpress Cell Culture Services (West Lebanon, New Hampshire, USA). Murine CTLA4Ig was a generous gift from Robert Peach (Bristol Myers Squibb, Princeton, New Jersey, USA). Anti-CD45RB mAb HB220 (American Type Culture Collection, Manassas, Virginia, USA) was purified on protein $\mathrm{G}$ columns according to the manufacturer's instructions (Pharmacia Biotech Inc., Piscataway, New Jersey, USA). The depleting anti-CD8 $\mathrm{mAb}$ ascites were prepared from hybridoma 2.43 (rat anti-mouse CD8) obtained from the American Type Culture Collection, as reported previously (25-27).

Transplantation. C57BL/6 mice (wild-type, IFN- $\gamma^{/-}$, $I L-2^{-/-}$, and $\mathrm{Bcl}-\mathrm{x}_{\mathrm{L}}$ transgenic) were used as recipients and $129 \mathrm{X} 1 / \mathrm{sv}, \mathrm{BALB} / \mathrm{c}$, and $\mathrm{C} 3 \mathrm{H} / \mathrm{He}$ mice were used as donors. The first cardiac allografts were placed in an intra-abdominal location $(22,28)$, and the second heart grafts from $\mathrm{BALB} / \mathrm{c}$ or $\mathrm{C} 3 \mathrm{H} / \mathrm{He}$ mice were placed in the neck using a modification of Chen's method (29). Graft function was assessed by daily palpation of heartbeat. Animals received either a single dose of CTLA4Ig ( $250 \mu \mathrm{g}$ on day 2 after transplant) or a four-dose protocol ( $250 \mu \mathrm{g}$ on day $0,2,4$, and 6$)$ by intraperitoneal injections. Anti-CD154 mAb was administered at a dose of $500 \mu \mathrm{g}$ intraperitoneally on day 0 and $250 \mu \mathrm{g}$ intraperitoneally on day 2, 4, and 6 after transplantation. Anti-CD45RB mAb was given intravenously as three doses of $100 \mu \mathrm{g}$ on day $-1,0$, and 5 after transplantation. These protocols were selected based on their proven efficacy $(22,30)$. Finally, in some experiments recipients received $0.1 \mathrm{ml}$ of unpurified ascites intraperitoneally (roughly equivalent to $100 \mu \mathrm{g}$ of purified $\mathrm{Ab}$ ) of the depleting anti-CD8 mAb (hybridoma 2.43 ) on days $-6,-3$, and -1 and weekly thereafter until week six after transplant. This vigorous course of $A b$ administration ensures greater than $95 \%$ depletion of the CD8 cells in the peripheral blood from the day of transplantation (25-27). The day of rejection was defined as the day in which cessation of palpable heartbeat occurred and was verified by autopsy and selective pathological examination. Loss of graft function within 48 hours of transplant was considered to be a technical failure ( $<10 \%$ on average), and these animals were excluded from further analysis.

Statistics. To evaluate graft survival, Kaplan-Meier survival graphs were constructed and log-rank com- 


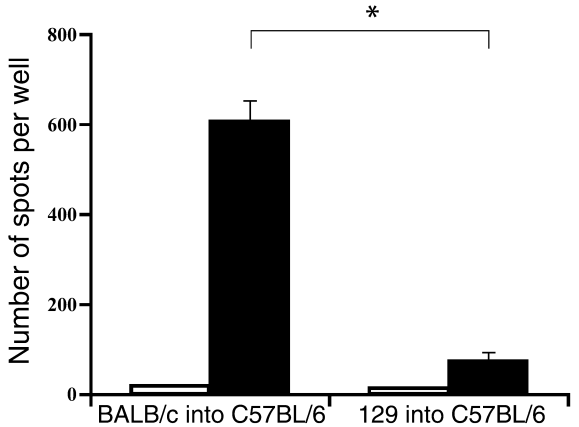

Figure 1

The frequency of IFN- $\gamma$-producing donor-specific T cells in C57BL/ 6 mice following allogeneic cardiac transplantation. Mice were transplanted with either MHC-mismatched BALB/c (left columns, $n=3$ ) or minor-mismatched $129 \times 1 /$ sv (right columns, $n=3$ ) allogeneic hearts. Recipient spleens were removed after rejection, and splenocytes $\left(1.2 \times 10^{6}\right.$ cells per well) were incubated with either recipient (white bars) or donor (black bars) irradiated splenocytes. The frequencies of IFN- $\gamma$-producing $T$ cells were then determined using ELISPOT assay as described in Methods. ${ }^{*} P=0.001$.

parison of the groups was used to calculate $P$ values. For the ELISPOT results $P$ values were calculated using the paired $t$ test.

\section{Results}

Analysis of alloreactive Tcell pool size against minor histocompatibility versus $M H C$ antigens. First, we investigated the relative frequency of alloreactive $\mathrm{T}$ cells responding to either MHC-mismatched or minor-mismatched alloantigens in vitro. After untreated $\mathrm{C} 57 \mathrm{BL} / 6$ recipients rejected MHC-mismatched plus multiple minor-mismatched BALB/c or only minor-mismatched $129 \mathrm{X} 1 / \mathrm{sv}$ cardiac allografts, they were sacrificed. Splenocytes from these mice were restimulated in vitro with irradiated donor-type splenocytes, and an ELISPOT assay was performed to estimate the frequency of IFN- $\gamma$-producing cells, as described (22). As shown in Figure 1, the frequency of alloreactive IFN- $\gamma$-producing recipient cells in response to $\mathrm{BALB} / \mathrm{c}$ stimulator cells was significantly higher (approximately ninefold) than that in response to $129 \mathrm{X} 1$ /sv stimulator cells.

While ELISPOT analysis is extremely sensitive in estimating the frequency of alloreactive $\mathrm{T}$ cells producing a particular cytokine ex vivo (22), the assay may not provide a direct measure of the frequency of alloreactive $\mathrm{T}$ cells in vivo. Therefore, we measured the comparative frequency of alloreactive precursor cells in vivo by using a parent-into- $\mathrm{F}_{1}$ adoptive transfer model, as described previously (24). CFSE-labeled C57BL/6 $\left(\mathrm{H}-2^{\mathrm{b}}\right)$ Thy $1.1^{+}$spleen and lymph node cells were transferred intravenously into either CB6F1 $\left(\mathrm{H}-2^{\mathrm{b} / \mathrm{d}}\right)$ or B6129F1 (H-2 $\left.{ }^{\mathrm{b}}\right)$ mice. Donor mice expressed the Thy1.1 allele, whereas recipient mice expressed Thy1.2, allowing donor $T$ cells to be identified after adoptive transfer by labeling with an anti-Thy $1.1 \mathrm{mAb}$. The proliferative response was analyzed 72 hours after adoptive transfer because this interval allows for optimal evalu- ation following multiple rounds of proliferation (24) (Figure 2a). As described previously (24), the responder frequency of alloreactive $\mathrm{T}$ cells was defined as the number of alloreactive precursor cells that divided at 72 hours as a percentage of the total number of donor cells recovered at that time point. Using this calculation, we found that the responder frequency for C57BL/ 6 cells reacting to MHC-mismatches in CB6F1 hosts was $8.01 \% \pm 1.15 \%$, whereas that for C $57 \mathrm{BL} / 6$ cells reacting to multiple minor mismatches only in B6129F1 hosts was $1.8 \% \pm 0.14 \%$. The proliferative capacity of alloreactive precursors also differed between the two strains: $10.9 \%$ of the responsive precursors divided at least five times in the MHC-mismatched CB6F1 strain, compared with $4.8 \%$ in the minor-mismatched B6129F1 strain. Since the number of donor cells that could be recovered at 72 hours was similar in donors responding to major or minor histocompatibility barriers, this suggests that there was no difference in the survival or homing to lymphoid organs in the two strains. Taken together, the in vitro ELISPOT and in vivo CFSE data show that the alloreactive $T$ cell clone size against minor-mismatched antigens is one-half to one log smaller than that against MHC-mismatched antigens in these models.

$\mathbf{a}$
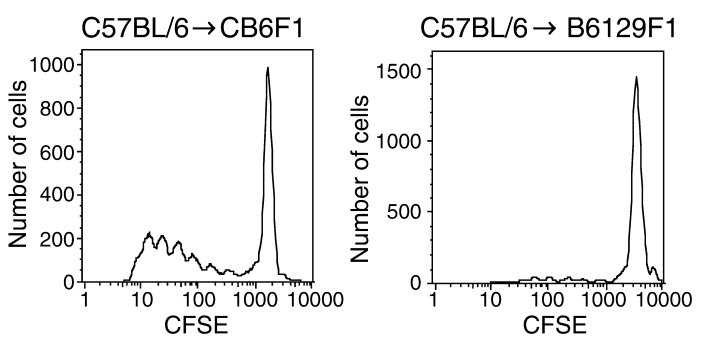

b
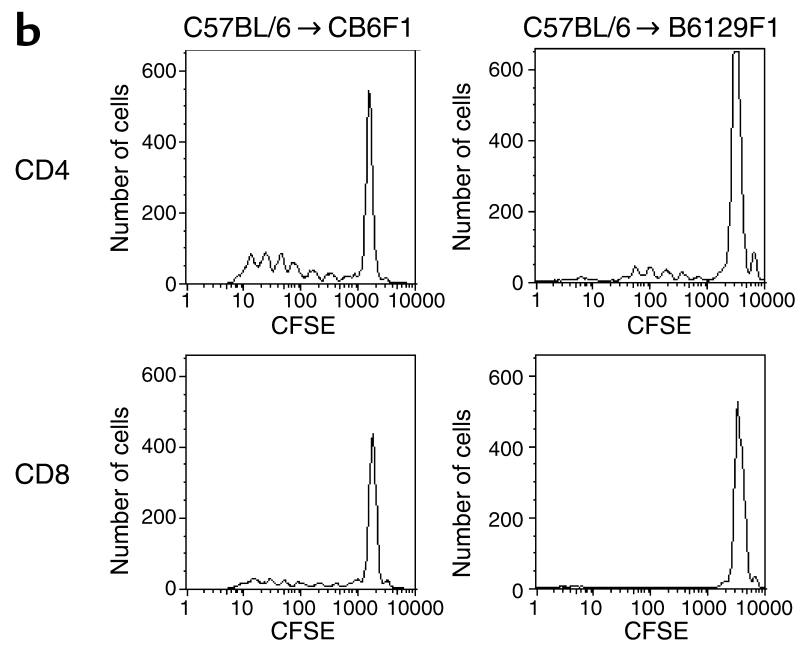

Figure 2

Determination of alloreactive T cell precursor frequency in vivo by CFSE. Flow-cytometry histograms gated on live CFSE-labeled C57BL/6-Thy1.1 cells $(\mathbf{a}), \mathrm{C} 57 \mathrm{BL} / 6-\mathrm{Thy} 1.1^{+} \mathrm{CD} 4^{+}$cells, or $\mathrm{C} 57 \mathrm{BL} / 6-\mathrm{Thy} 1.1^{+} \mathrm{CD} 8^{+}$cells (b) recovered 72 hours after adoptive transfer into CB6F1 or B6129F1 recipients. The rightmost peak on each graph represents undivided cells. Data are representative of four experiments. 
a MHC-mismatched combination

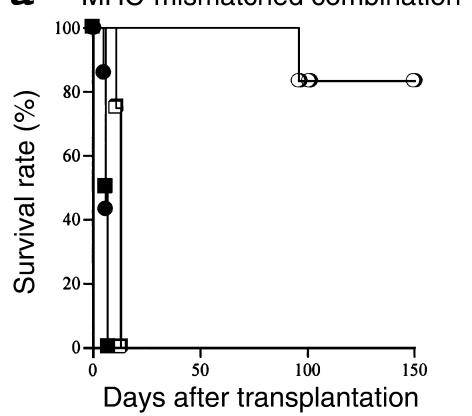

b Minor-mismatched combination

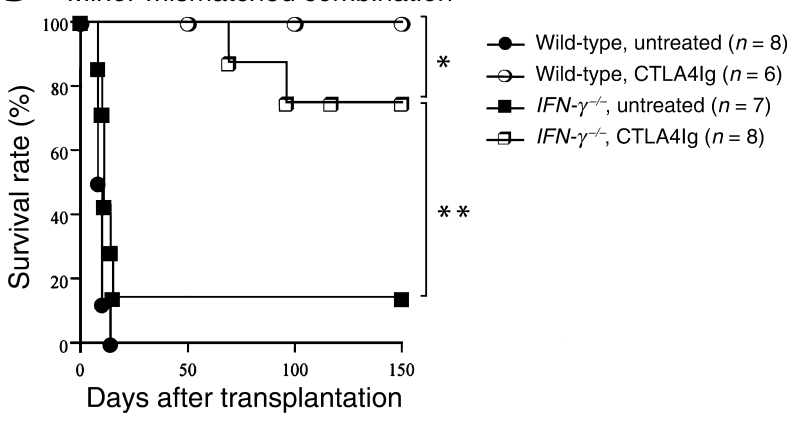

Figure 3

The effect of endogenous IFN- $\gamma$ on long-term cardiac allograft survival induced by $\mathrm{CD} 28-\mathrm{B} 7$ blockade in a MHC-mismatched (BALB/C into C57BL/6) combination (a) or a minor-mismatched (129 into C57BL/6) combination (b). Wild-type or IFN- $\gamma^{/-}$recipients were treated with or without CTLA4Ig ( $250 \mu \mathrm{g}$ intraperitoneally on days $0,2,4$, and 6 after transplantation) as described in Methods. ${ }^{*} P=0.205 ;{ }^{*} P=0.003$.

Effect of CD28-B7 blockade on MHC-mismatched versus minor-mismatched cardiac allograft rejection in $I F N-\gamma^{\prime-}$ recipients. Given the difference in alloreactive $\mathrm{T}$ cell clone size shown above, we investigated the effect of CD28-B7 blockade by CTLA4Ig in the two (MHC-mismatched versus minor-mismatched) donor-recipient combinations in recipients that lacked IFN- $\gamma$ (Figure 3). Wildtype or $I F N-\gamma^{/-}$C57BL/6 mice were transplanted with $\mathrm{BALB} / \mathrm{c}$ or $129 \mathrm{X} 1 / \mathrm{sv}$ cardiac allografts and treated with or without CTLA $4 \operatorname{Ig}(250 \mu \mathrm{g}$ on days $0,2,4$, and 6 after transplant). In the MHC-mismatched combination, the survival time of cardiac allografts transplanted into untreated IFN- $\gamma^{-}$mice was comparable to that observed in untreated wild-type recipients $\left(I F N-\gamma^{\prime-}\right.$ recipients: mean survival time [MST] $6.50 \pm 0.33$ days, $n=4$; wildtype recipients: MST $6.28 \pm 0.57$ days, $n=7, P=0.70$ ). However, while CTLA4Ig induced long-term survival in wild-type recipients of MHC-mismatched allografts, this treatment failed to do so in the $\mathrm{IFN}-\gamma^{\prime-}$ group (Figure $3 a)$. In the minor-mismatched combination, engraftment of cardiac allografts was significantly prolonged in untreated $I F N-\gamma^{\prime-}$ as compared with wild-type recipients, primarily due to the occurrence of long-term functioning grafts in few untreated recipients (Figure $3 \mathrm{~b}$ ). However, in contrast to the MHC-mismatched combination, CTLA4Ig promoted long-term allograft survival (Figure $3 \mathrm{~b}$ ) and donor-specific tolerance (Table 1) in both wild-type and $I F N-\gamma^{\prime-}$ recipients of minor-mis- matched grafts. These data indicate that while IFN- $\gamma$ is necessary for induction of tolerance by CTLA4Ig in conditions of high alloreactive T cell clone size (MHC-mismatched combination), IFN- $\gamma$ is not necessary for tolerance induction when the alloreactive $\mathrm{T}$ cell clone size is relatively small (minor-mismatched combination).

Effect of CD154-CD40 blockade, anti-CD45RB, or combination strategies on MHC-mismatched versus minor-mismatched cardiac allograft rejection in $I F N-\gamma^{-/}$recipients. To determine if the differential effects of MHC mismatching versus minor antigens mismatching on the ability to induce long-term allograft survival in $I F N-\gamma^{-}$recipients was unique to CD28-B7 T cell costimulatory blockade or could be seen with other tolerance-induction protocols, we investigated other strategies including CD154-CD40 blockade. In the MHC-mismatched combination, similar to our findings with CTLA4Ig, while treatment with anti-CD154 $\mathrm{mAb}$ induced long-term survival in wild-type recipients of the MHC-mismatched cardiac allograft, it failed to do so in $I F N-\gamma^{\prime-}$ recipients (Figure $4 a)$. In the minor-mismatched combination, anti$\mathrm{CD} 154 \mathrm{mAb}$ treatment prolonged cardiac allograft survival indefinitely and induced donor-specific tolerance in wild-type recipients (Figure $4 \mathrm{~b}$ and Table 1). However, unlike CTLA4Ig, only $57 \%$ of $I F N-\gamma^{\prime-}$ recipients treated with anti-CD154 mAb exhibited long-term survival of minor-mismatched allografts, although there was no statistically significant difference between wildtype and $I F N-\gamma^{\prime-}$ recipients (Figure $4 \mathrm{~b}$ ).

Since alteration of signal 1 created by treatment with anti-CD45RB mAb induces long-term graft survival in MHC-mismatched allografts (31), we investigated whether the tolerogenic effect of anti-CD45RB mAb also requires IFN- $\gamma$ using either MHC-mismatched or minor-mismatched grafts. In the MHC-mismatched combination, although anti-CD45RB mAb significantly prolonged cardiac allograft survival in wild-type recipients, treated $I F N-\gamma^{\prime-}$ recipients rejected their grafts at the same tempo as untreated $I F N-\gamma /$ recipients (Figure $5 \mathrm{a})$. In contrast, anti-CD45RB mAb was equally effective in prolonging allograft survival in wild-type and $I F N-\gamma^{-}$ recipients receiving MHC-matched, minor-mismatched grafts (Figure $5 \mathrm{~b}$ ). These findings suggest that, similar to $T$ cell costimulatory blockade, graft prolongation with signal 1 alteration requires IFN- $\gamma$ in MHC-mismatched but not minor-mismatched combinations.

\section{Table 1}

Donor-specific tolerance in minor-mismatched combinations: second heart graft survival (days) in animals bearing original heart grafts for more than 100 days

\begin{tabular}{ccccc}
\cline { 3 - 4 } Donor & Recipient & Treatment & \multicolumn{2}{c}{ Second graft donor } \\
& & & 129 & $\mathrm{C} 3 \mathrm{H} / \mathrm{He}$ \\
129 & Wild-type & CTLA4Ig & $>56,>100,>100$ & 6,6 \\
129 & IFN- $\gamma^{\prime-}$ & CTLA4Ig & $>100,>100,>100$ & 7 \\
129 & Wild-type & Anti-CD154 & $20,>90,>90$ & 6,29 \\
\hline
\end{tabular}


a MHC-mismatched combination
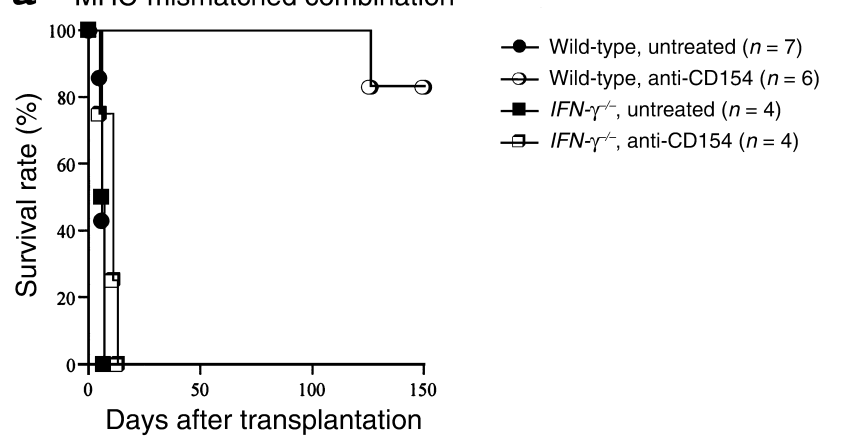

b Minor-mismatched combination

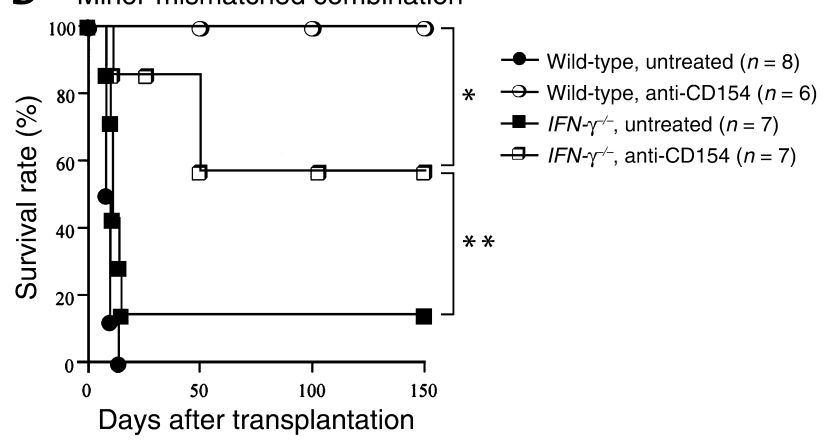

Figure 4

The effect of endogenous IFN- $\gamma$ on long-term cardiac allograft survival induced by CD154-CD40 blockade in a MHC-mismatched (BALB/c into $\mathrm{C} 57 \mathrm{BL} / 6$ ) combination (a) or a minor-mismatched (129 into C57BL/6) combination (b). Wild-type or IFN- $\gamma^{\prime-}$ recipients were treated with or without anti-CD154 mAb (administered at a dose of $500 \mu \mathrm{g}$ intraperitoneally on day 0 and $250 \mu \mathrm{g}$ on day 2,4 , and 6 after transplantation) as described in Methods. ${ }^{*} P=0.062 ;{ }^{*} P=0.029$.

We and others have demonstrated previously that simultaneous blockade of CD28-B7 and CD154-CD40 pathways or blockade of CD154-CD40 plus CD45RB modulation is extremely effective in promoting longterm allograft survival and tolerance, especially in stringent transplant models $(30,32)$. Therefore, we investigated the role of IFN- $\gamma$ in tolerance induction across MHC barriers using two powerful tolerogenic strategies: either CTLA4Ig combined with anti-CD154 mAb (32) or anti-CD154 mAb combined with anti-CD45RB mAb (30). As shown in Figure 6, although both combination strategies provide for significantly prolonged graft survival as compared with results achieved with a single agent alone, all grafts were rejected within 4 weeks in $I F N-\gamma^{\prime-}$ recipients. These findings confirm resistance to tolerance in MHC-mismatched combinations in the absence of IFN- $\gamma$, even with therapeutic strategies that are extremely effective in stringent models.

Role of alloreactive $C D 8^{+} T$ cells. In an interesting study, Larsen's group suggested that alloreactive $\mathrm{CD}^{+} \mathrm{T}$ cells may play an important role in costimulatory pathway blockade-resistant rejection in some strain combinations $(33,34)$. Therefore, we examined the possibility that the difference of survival time between MHC and minormismatched allografts transplanted into $I F N-\gamma^{\prime-}$ recipients may result from the differences in $\mathrm{CD}^{+}$alloreactive
$\mathrm{T}$ cell clone size and/or effector functions in recipients of MHC versus minor-mismatched allografts. First, we measured the frequency of alloreactive $\mathrm{CD}^{+}$versus $\mathrm{CD}^{+} \mathrm{T}$ cells in wild-type animals in response to $\mathrm{MHC}$ or multiple minor antigens using the same CFSE assay described above. As shown in Figure 2b, responder frequencies for $\mathrm{CD}^{+}$versus $\mathrm{CD} 8^{+} \mathrm{T}$ cells reacting to $\mathrm{MHC}$ mismatches were $10.2 \% \pm 1.6 \%$ versus $5.4 \% \pm 1.5 \%$, respectively, while those reacting to multiple minor mismatches were $3.0 \% \pm 0.3 \%$ versus $1.3 \% \pm 0.3 \%$, respectively. These data show that there were no major differences in the responding $\mathrm{CD}^{+} / \mathrm{CD}^{+} \mathrm{T}$ cell ratio for $\mathrm{MHC}$ (ratio around 1.9) and multiple minor mismatches (ratio around 2.3), indicating that variations in the relative contributions of these two subsets to the alloreactive response was unlikely to account for differences in tolerance susceptibility in MHC versus minor mismatches. However, due to the greater overall numbers of cells reactive to MHC-mismatched antigens, the absolute frequency of alloreactive $\mathrm{CD}^{+} \mathrm{T}$ cells was approximately four times higher against MHC antigens than that against minor antigens. Given the known role of $\mathrm{CD}^{+}$ cells in costimulatory blockade-resistant rejection in certain models $(33,34)$ and the functions of $\mathrm{CD}^{+} \mathrm{T}$ cells in allograft rejection in $I F N-\gamma^{\prime-}$ recipients (35), we sought to further address the potential role that effector $\mathrm{CD}^{+}$ $\mathrm{T}$ cells may play in resistance to tolerance in the fully

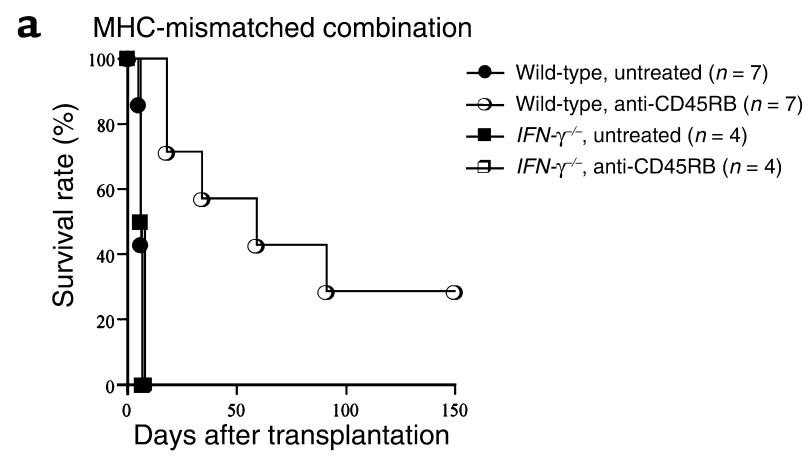

b Minor-mismatched combination

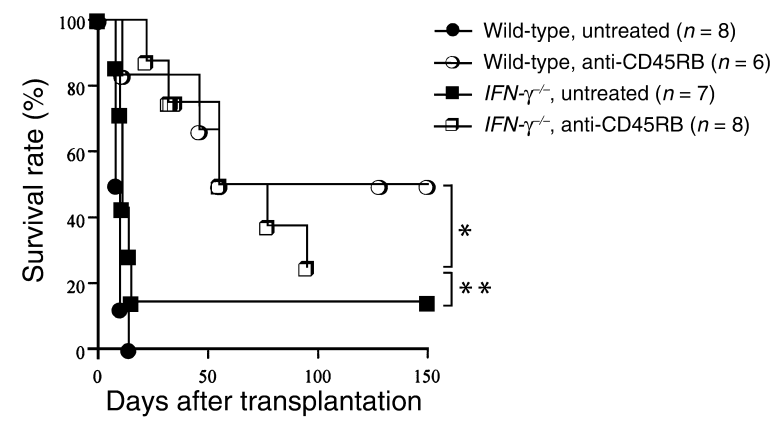

Figure 5

The effect of endogenous IFN- $\gamma$ on long-term cardiac allograft survival induced by CD45RB blockade in a MHC-mismatched (BALB/c into C57BL/6) combination (a) or a minor-mismatched (129 into $\mathrm{C} 57 \mathrm{BL} / 6)$ combination (b). Wild-type or IFN- $\gamma^{-/}$recipients were treated with or without anti-CD45RB mAb (given intravenously as three doses of $100 \mu \mathrm{g}$ on day $-1,0$, and 5 after transplantation) as described in Methods. ${ }^{*} P=0.240 ;{ }^{*} P=0.039$. 
a MHC-mismatched combination

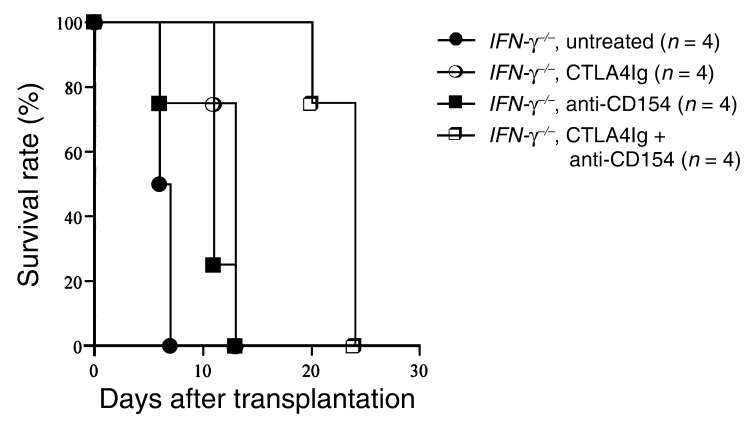

b MHC-mismatched combination

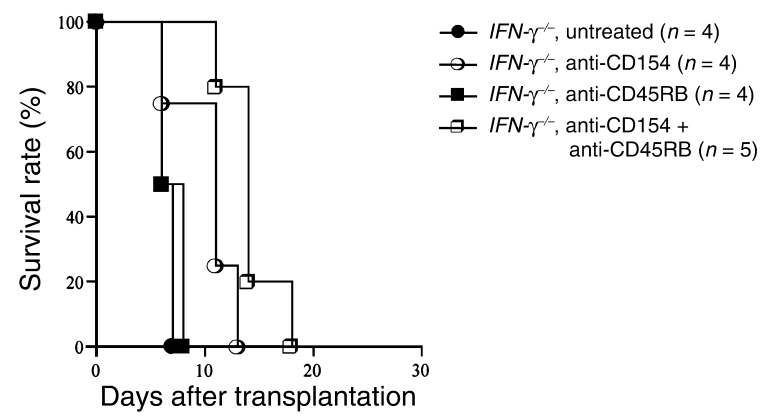

\section{Figure 6}

The effect of endogenous IFN- $\gamma$ on long-term cardiac allograft survival in a MHC-mismatched (BALB/c into C57BL/6) combination. $I F N-\gamma^{\prime-}$ recipients were treated with coadministration of CTLA4Ig + anti-CD154 mAb (a), or with coadministration of anti-CD154 mAb + anti-CD45RB mAb (b) as described in Methods. $P$ values are as follows: CTLA4Ig alone vs. CTLA4Ig + anti-CD154, $P=0.01$; antiCD154 alone vs. CTLA4Ig + anti-CD154, $P=0.007$; anti-CD154 alone vs. anti-CD154 + anti-CD45RB, $P=0.019$; anti-CD45RB alone vs. anti-CD154 + anti-CD45RB, $P=0.004$.

allogeneic combination in IFN- $\gamma$-deficient mice. Therefore, we vigorously depleted $\mathrm{CD} 8^{+} \mathrm{T}$ cells (see Methods) from $I F N-\gamma^{\prime-}$ recipients of fully allogeneic cardiac allografts treated with a protocol of CTLA4Ig plus MR1 (the protocol that gave the best fully allogeneic allograft survival in $I F N-\gamma^{\prime-}$ recipients; see results above and Figure 6a). Although we experienced early death (before day 10) with functioning grafts of a few animals for unclear reasons, surviving recipients $(n=4)$ exhibited significant $(P=0.01, \log$-rank test) prolongation of graft survival (all grafts are surviving > 60 days) when compared with the combined costimulation blockade group alone (all animals rejected within 25 days; see Figure 6a). Collectively, these data strongly support the hypothesis that resistance to tolerance in MHC-mismatched combinations in the absence of IFN- $\gamma$ results from the larger donor-reactive $\mathrm{T}$ cell clone size, especially in the $\mathrm{CD}^{+} \mathrm{T}$ cell compartment, compared with the minor-mismatched combination in which tolerance is readily inducible.

Effect of CD28-B7 blockade on minor-mismatched cardiac allograft rejection in $\mathrm{IL}-2^{-/-}$recipients and Bcl- $x_{L}$ transgenic animals. We next sought to extend our observations to other models of defective T cell apoptosis. Previously, we and others have shown that IL-2, similar to IFN- $\gamma$, is necessary for tolerance induction by $\mathrm{T}$ cell costimulatory blockade across MHC barriers, presumably by promoting AICD of alloreactive T cell clones $(10,11,17)$. Therefore, we next examined whether or not IL-2 was required for tolerance induction in conditions of small, alloreactive $\mathrm{T}$ cell clone size in minor-mismatched combination. We used CTLA4Ig for tolerance induction because this treatment reliably produced tolerance in our models (see above). Similar to our observation in $I F N-\gamma^{\prime-}$ recipients, and as reported previously, acute MHC-mismatched cardiac allografts were rejected within 15 days in untreated $I L-2^{-/-}$recipients (Figure $7 \mathrm{a})$, indicating that these cytokines play a redundant role in MHC-mismatched cardiac allograft rejection $(36,37)$. When treated with CTLA4Ig, MHC-mismatched cardiac allografts in $I L-2^{-/-}$recipients had modestly increased survival compared with untreated $I L-2^{-/-}$animals (Figure 7a), although all of them were rejected by day 40 after transplant $(P=0.010)$. In contrast, CTLA4Ig treatment induced long-term survival of minor-mismatched allografts in $I L-2^{-/-}$recipients as well as wild-type animals (Figure $7 b$ ).

Finally, our group has shown previously that passive $\mathrm{T}$ cell death plays an important role in tolerance induction by $\mathrm{T}$ cell costimulatory blockade, because CTLA4Ig and anti-CD154 mAb therapy failed to tolerize Bcl- $\mathrm{x}_{\mathrm{L}}$ transgenic recipients of MHC-mismatched grafts (11). Therefore, we used Bcl- $x_{L}$ transgenic animals to determine whether passive cell death is indeed required under conditions of small alloreactive $T$ cell clone size in a minor-mismatched combination. Interestingly, untreated $\mathrm{Bcl}-\mathrm{x}_{\mathrm{L}}$ transgenic recipients had significantly worse cardiac allograft survival than littermate controls, with $10-20 \%$ of littermate control animals showing spontaneous long-term (>100 days) engraftment. A single injection of CTLA4Ig on day 2 after transplant induced long-term allograft survival in both wild-type and Bcl- $x_{\mathrm{L}}$ transgenic animals (Figure 8a). Donor-specific tolerance was confirmed in the $\mathrm{Bcl}-\mathrm{x}_{\mathrm{L}}$ transgenic animals by acceptance of a second set of grafts from the original donor (graft survival $>100$ days, $n=2$ ) and rejection of third-party grafts (graft survival 5 and 7 days). Consistent with our previous report (11), a similar protocol of CTLA4Ig was relatively ineffective in promoting long-term allograft survival across MHC barriers (data not shown). However, when we used multiple doses of CTLA4Ig (250 $\mu \mathrm{g}$ on day $0,2,4$, and 6), both wild-type and $\mathrm{Bcl}-\mathrm{x}_{\mathrm{L}}$ transgenic recipients had prolonged graft survival, but all Bcl- $\mathrm{x}_{\mathrm{L}}$ transgenic recipients ultimately rejected their allografts (Figure 8b). These data indicate that while passive $T$ cell death is required for induction of tolerance by costimulatory blockade across MHC barriers, it is not necessary for tolerance induction in the minor-mismatched combination.

\section{Discussion}

The primary findings of this study are that induction of peripheral transplantation tolerance across MHC barriers requires the presence of the Th1 cytokines, 
a MHC-mismatched combination

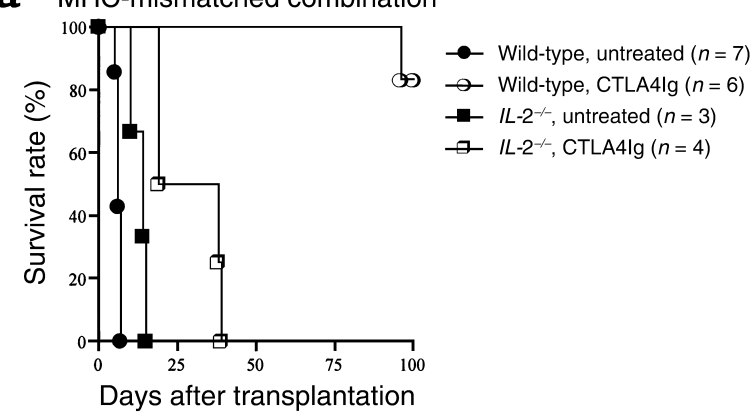

b Minor-mismatched combination

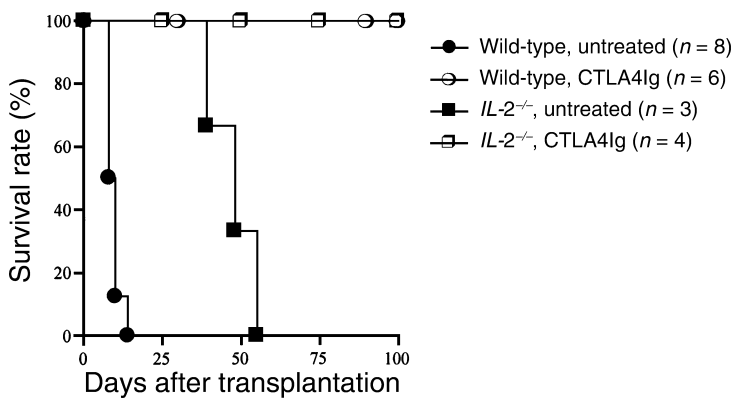

Figure 7

The effect of endogenous IL-2 on long-term cardiac allograft survival induced by CD28-B7 blockade in a MHC-mismatched (BALB/c into C57BL/6) combination (a) or a minor-mismatched (129 into $\mathrm{C} 57 \mathrm{BL} / 6)$ combination (b). Wild-type or IFN- $\gamma^{/-}$recipients were treated with or without CTLA4Ig as described in Methods.

IFN- $\gamma$ and IL-2, and depends upon intact susceptibility of recipient $\mathrm{T}$ cells to undergo programmed cell death, while this is not required for tolerance induction across multiple minor histocompatibility mismatches.

Reduction of the alloreactive $T$ cell clone mass by limiting proliferation and by programmed cell death appears to be required for the induction of peripheral tolerance to fully allogeneic transplants by $\mathrm{T}$ cell costimulatory blockade $(8,10-12,38)$. In this study we tested the hypothesis that this requirement may not be the case in conditions where the alloreactive $\mathrm{T}$ cell clone size is relatively small, such as in a minor-mismatched combination. Indeed, we show by in vitro ELISPOT and in vivo CFSE analyses that the alloreactive $T$ cell clone size against minor-mismatched antigens was approximately four- to ninefold less than that against MHC-mismatched antigens. Furthermore, we demonstrate here, we believe for the first time, that CD28-B7 T cell costimulatory blockade does not require both of the prototypical Th1 cytokines, IL- 2 and IFN- $\gamma$, nor intact mechanisms of passive cell death to achieve transplant tolerance across minor histocompatibility barriers. These findings suggest that unlike the MHC-mismatched combination, tolerance induction across minor histocompatibility antigens does not require programmed death of alloreactive $\mathrm{T}$ cells.

Why might there be differential requirements for $\mathrm{T}$ cell death in tolerance as a function of the size of the responding $\mathrm{T}$ cell pool? Clearly, regulatory mechanisms evolve normally in the course of immune responses and are critical to prevent autoimmunity (7). Regulatory cells have a role in the maintenance phase of peripheral tolerance strategies, presumably to neutralize new thymic emigrants. Our previous work showing a need for $\mathrm{T}$ cell programmed cell death for the long-term success of peripheral tolerance approaches argued that death of alloreactive $T$ cells during the induction phase of tolerance was required to limit the size of the alloreactive $T$ cell clone mass $(11,17)$. In the absence of this constraint on the alloreactive $\mathrm{T}$ cell mass, regulatory cells may be unable to control the allograft response leading to failure of tolerance induction $(12,17,39,40)$. In contrast, in autoimmune models the much smaller pool of antigen-reactive $T$ cells could be controlled through regulatory cells and/or Th2 immune deviation alone $(18,19)$ and did not require "clonal reduction" through programmed cell death for the costimulatory blockade to be effective (ref. 41 and our unpublished observations). Consistent with this finding, our group has also shown that immune deviation from Th1 to Th2 alone by neutralizing anti-IL- $12 \mathrm{mAb}$ therapy can induce transplantation tolerance across minor histocompatibility barriers but fails to do so across fully allogeneic barriers (20). Therefore, our model system predicts that under conditions where the alloreactive $\mathrm{T}$ cell clone size is smaller than in MHC-mismatched

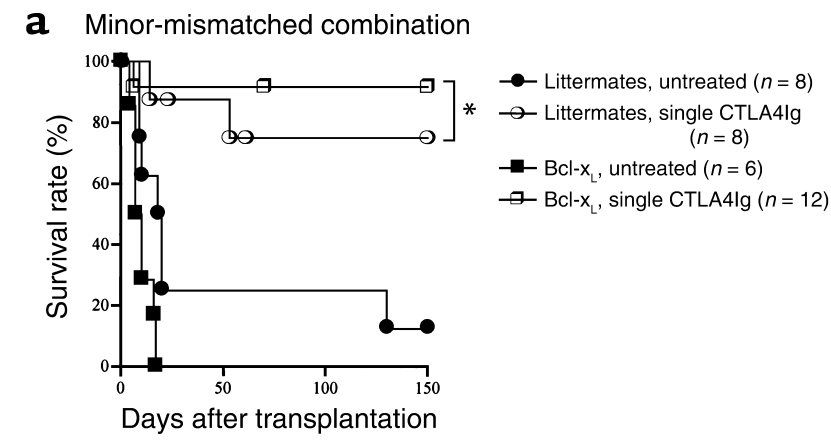

b MHC-mismatched combination

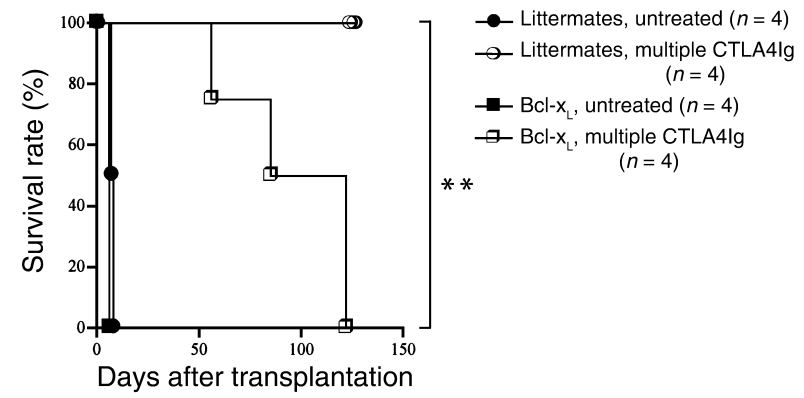

Figure 8

The effect of passive cell death on long-term cardiac allograft survival induced by CD28-B7 blockade in a minor-mismatched (129 into C57BL/6) combination (a) or a MHC-mismatched (BALB/c into $\mathrm{C} 57 \mathrm{BL} / 6$ ) combination (b). Bcl-x transgenic or their littermate recipients were treated with or without either a single dose (a) or four doses (b) of CTLA4Ig as described in Methods. ${ }^{*} P=0.327 ;{ }^{*} P=0.008$. 
grafts, tolerance by costimulatory blockade may be achievable even in animals with deficient $\mathrm{T}$ cell apoptotic pathways. The experiments reported in this paper validate this prediction and support our hypothesis. As noted above, increasing data suggest that regulatory cells are required in the maintenance phase of tolerance (42-45). These cells, as generated in infectious tolerance, were first described in minor histocompatibility-mismatched systems and not in MHC-mismatched ones (46). Recently, infectious tolerance has been reported to prevent graft rejection across an MHC mismatch $(42,47)$. This required the adoptive transfer of a large number (50 million) of regulatory cells into the recipient mouse. This elegant experiment demonstrates that regulatory cells are inherently capable of inducing as well as maintaining tolerance, although the sheer number of them required to do so may explain why experiments designed to use in vivo immune deviation to tolerize across an MHC barrier have generally failed. Moreover, the expansion of large numbers of regulatory cells in vivo may require the death of other cytopathic cells in the primary host (Strom et al., unpublished data). The lesson we draw from these systems is that the capacity of therapeutic interventions to induce tolerance is tightly associated with the size of the antigen-reactive $\mathrm{T}$ cell clone size.

Our experiments used three distinct models to test the requirements for limiting $\mathrm{T}$ cell expansion and $\mathrm{T}$ cell apoptosis in the induction of transplantation tolerance: IFN- $\gamma$-deficient mice, IL-2-deficient mice, and $\mathrm{Bcl}-\mathrm{x}_{\mathrm{L}}$ transgenic mice. While we favor the interpretation that the contrasting findings we observed in MHC-mismatched versus minor-mismatched combinations are due to differences in the size of the alloreactive $\mathrm{T}$ cell clones in those situations, we recognize that other important differences exist as well. However, the consistency of our observations across all three models tested emphasizes the fundamental importance of our findings and the likelihood that the outcome of the allograft response in a given host, rejection or tolerance, depends in part upon the size of the T cell pool able to mount a graft-destructive response. Additional support of our conclusions comes from the observation that a relationship between clone size and tolerance does exist in a model system in which systemic administration of antigen in vivo leads to peripheral deletion of most of the of the peptide-specific $T$ cells, with the few remaining residual cells rendered anergic (48). In this system, the residual nondeleted $\mathrm{T}$ cells are unable to provide $\mathrm{B}$ cell help for $\mathrm{Ab}$ production. If the pool of hyporesponsive $\mathrm{T}$ cells is increased by transferring large numbers of cells from tolerized donors into naive recipients, $\mathrm{B}$ cell help and Ab production is restored (49). We do realize, however, that alternative explanations need to be considered. It is possible that the differences we observed in the major versus minor combinations in our model may be due to the differential role of $\mathrm{CD}^{+}$versus $\mathrm{CD}^{+} \mathrm{T}$ cells in the rejection process, the differences in the numbers and types of cells expressing the alloantigen, the density of the alloantigen on the surface of those cells, and the contribution of direct versus indirect allorecognition pathways to rejection and tolerance. Our experiments measuring the frequencies of alloreactive $\mathrm{CD}^{+}$ and $\mathrm{CD}^{+} \mathrm{T}$ cells by CFSE analysis indicate that the relative $\mathrm{CD} 4 / \mathrm{CD} 8$ ratios of responding cells were not significantly different between major and minor combinations. However, our in vivo studies with vigorous CD8 depletion in $I F N-\gamma^{-}$recipients of fully mismatched grafts, taken together with the rest of our in vivo data described above, suggest that alloreactive $\mathrm{CD}^{+} \mathrm{T}$ cells, or a major component of that alloreactive $\mathrm{T}$ cell pool, must undergo apoptotic cell death to achieve tolerance. In recipients of minor antigen-mismatched grafts, $\mathrm{T}$ cell costimulatory blockade alone appears to be sufficient to control the relatively small alloreactive $\mathrm{CD}^{+}$and $\mathrm{CD}^{+} \mathrm{T}$ cell pool resulting in long-term graft survival and tolerance even in recipients that are defective in $\mathrm{T}$ cell apoptosis. Ongoing studies in our laboratory are designed to further dissect the mechanisms of the differential requirements for tolerance induction in recipients of fully allogeneic versus minor-mismatched allografts.

In conclusion, the requirement for Th1 cytokines and $T$ cell death for induction of transplantation tolerance is not universal; the alloreactive T cell clone size, especially the alloreactive $\mathrm{CD}^{+} \mathrm{T}$ cell pool, appears to be an important determinant for this requirement. Our data have implications for the design and implementation of clinical strategies to induce transplantation tolerance in humans and suggest that the immunomodulatory or tolerogenic regimen may need to be tailored according to the degree of antigen matching between donor and recipient (50).

\section{Acknowledgments}

This work was supported by NIH research grants RO1AI34965 (to M.H. Sayegh), PO1-AI41521 (to M.H. Sayegh, L.A. Turka, and T.B. Strom), and RO1-AI45485 (to D.M. Rothstein). We thank Akira Yamada for his technical assistance.

1. Lenardo, M.J. 1991. Interleukin-2 programs mouse alpha beta T lymphocytes for apoptosis. Nature. 353:858-861.

2. Boehme, S.A., and Lenardo, M.J. 1993. Propriocidal apoptosis of mature $\mathrm{T}$ lymphocytes occurs at $\mathrm{S}$ phase of the cell cycle. Eur. J. Immunol. 23:1552-1560.

3. Critchfield, J.M., et al. 1994. T cell deletion in high antigen dose therapy of autoimmune encephalomyelitis. Science. 263:1139-1143.

4. Dalton, D.K., Haynes, L., Chu, C.Q., Swain, S.L., and Wittmer, S. 2000. Interferon gamma eliminates responding CD4 T cells during mycobacterial infection by inducing apoptosis of activated CD4 T cells. J. Exp. Med. 192:117-122.

5. Chu, C.Q., Wittmer, S., and Dalton, D.K. 2000. Failure to suppress the expansion of the activated CD4 T cell population in interferon gammadeficient mice leads to exacerbation of experimental autoimmune encephalomyelitis. J. Exp. Med. 192:123-128.

6. Badovinac, V.P., Tvinnereim, A.R., and Harty, J.T. 2000. Regulation of antigen-specific CD8(+) T cell homeostasis by perforin and interferongamma. Science. 290:1354-1358.

7. Van Parijs, L., and Abbas, A.K. 1998. Homeostasis and self-tolerance in the immune system: turning lymphocytes off. Science. 280:243-248.

8. Hassan, A.T., et al. 1999. Regulation of alloantigen-mediated T-cell proliferation by endogenous interferon-gamma: implications for long-term allograft acceptance. Transplantation. 68:124-129. 
9. Li, X.C., et al. 2001. IL-15 and IL-2: a matter of life and death for T cells in vivo. Nat. Med. 7:114-118.

10. Dai, Z., Konieczny, B.T., Baddoura, F.K., and Lakkis, F.G. 1998. Impaired alloantigen-mediated $\mathrm{T}$ cell apoptosis and failure to induce long-term allograft survival in IL-2-deficient mice. J. Immunol. 161:1659-1663.

11. Wells, A.D., et al. 1999. Requirement for T-cell apoptosis in the induction of peripheral transplantation tolerance. Nat. Med. 5:1303-1307.

12. Li, X.C., et al. 1999. Induction of allograft tolerance in the absence of Fasmediated apoptosis. J. Immunol. 163:2500-2507.

13. Sayegh, M.H., and Turka, L.A. 1998. The role of T-cell costimulatory activation pathways in transplant rejection. N. Engl. J. Med. 338:1813-1821.

14. Kishimoto, K., Dong, V.M., and Sayegh, M.H. 2000. The role of costimulatory molecules as targets for new immunosuppressives in transplantation. Curr. Opin. Urol. 10:57-62.

15. Markees, T.G., et al. 1998. Long-term survival of skin allografts induced by donor splenocytes and anti-CD154 antibody in thymectomized mice requires CD4(+) T cells, interferon-gamma, and CTLA4. J. Clin. Invest. 101:2446-2455.

16. Konieczny, B.T., et al. 1998. IFN-gamma is critical for long-term allograft survival induced by blocking the CD28 and CD40 ligand T cell costimulation pathways. J. Immunol. 160:2059-2064.

17. Li, Y., et al. 1999. Blocking both signal 1 and signal 2 of T-cell activation prevents apoptosis of alloreactive $\mathrm{T}$ cells and induction of peripheral allograft tolerance. Nat. Med. 5:1298-1302.

18. Chen, Y., Kuchroo, V.K., Inobe, J.-I., Hafler, D.A., and Weiner, H.L. 1994 Regulatory $\mathrm{T}$ cell clones induced by oral tolerance: Suppression of autoimmune encephalomyelitis. Science. 265:1237-1240.

19. Khoury, S.J., et al. 1995. CD28-B7 costimulatory blockade by CTLA4Ig prevents actively induced experimental autoimmune encephalomyelitis and inhibits Th1 but spares Th2 cytokines in the central nervous system. J. Immunol. 155:4521-4524.

20. Li, X.C., Zand, M.S., Li, Y., Zheng, X.X., and Strom, T.B. 1998. On histocompatibility barriers, Th1 to Th2 immune deviation, and the nature of the allograft responses. J. Immunol. 161:2241-2247.

21. Benichou, G., Valujskikh, A., and Heeger, P.S. 1999. Contributions of direct and indirect $\mathrm{T}$ cell alloreactivity during allograft rejection in mice. J. Immunol. 162:352-358.

22. Kishimoto, K., et al. 2000. The role of CD154-CD40 versus CD28-B7 costimulatory pathways in regulating allogeneic Th 1 and $T h 2$ responses in vivo. J. Clin. Invest. 106:63-72.

23. Wells, A.D., Gudmundsdottir, H., and Turka, L.A. 1997. Following the fate of individual $\mathrm{T}$ cells throughout activation and clonal expansion. Signals from $T$ cell receptor and CD28 differentially regulate the induction and duration of a proliferative response. J. Clin. Invest. 100:3173-3183.

24. Suchin, E.J., et al. 2001 Quantifying the frequency of alloreactive T cells in vivo: new answers to an old question. J. Immunol. 166:973-981.

25. Ghobrial, R.R., Boublik, M., Winn, H.J., and Auchincloss, H., Jr. 1989. In vivo use of mAb's against murine $\mathrm{T}$ cell antigens. Clin. Immunol. Immunopathol. 52:486-506.

26. Yamada, A., et al. 2001. Recipient MHC class II expression is required to achieve long-term survival of murine cardiac allografts after costimulatory blockade. J. Immunol. 167:5522-5526.

27. Yamada, A., et al. 2001. CD28-independent costimulation of T cells in alloimmune responses. J. Immunol. 167:140-146.

28. Corry, R.J., Winn, H.J., and Russell, P.S. 1973. Primarily vascularized allografts of hearts in mice. The role of $\mathrm{H}-2 \mathrm{D}, \mathrm{H}-2 \mathrm{~K}$, and non- $\mathrm{H}-2$ antigens in rejection. Transplantation. 16:343-350.
29. Chen, Z.H. 1991. A technique of cervical heterotopic heart transplantation in mice. Transplantation. 52:1099-1101.

30. Rothstein, D.M., et al. 2001. Targeting signal 1 through CD45RB synergizes with CD40 ligand blockade and promotes long term engraftment and tolerance in stringent transplant models. J. Immunol. 166:322-329.

31. Lazarovits, A.I., et al. 1996. Prevention and reversal of renal allograft rejection by antibody against CD45RB. Nature. 380:717-720.

32. Larsen, C.P., et al. 1996. Long-term acceptance of skin and cardiac allografts after blocking CD40 and CD28 pathways. Nature. 381:434-438.

33. Trambley, J., et al. 1999. Asialo GM1(+) CD8(+) T cells play a critical role in costimulation blockade-resistant allograft rejection. J. Clin. Invest. 104:1715-1722.

34. Williams, M.A., et al. 2000. Genetic characterization of strain differences in the ability to mediate CD40/CD28-independent rejection of skin allografts. J. Immunol. 165:6849-6857.

35. Bishop, D.K., Wood, S.C., Eichwald, E.J., and Orosz, C.G. 2001. Immunobiology of allograft rejection in the absence of IFN-gamma: CD8+ effector cells develop independently of CD4+ cells and CD40-CD40 ligand interactions. J. Immunol. 166:3248-3255.

36. Saleem, S., Konieczny, B.T., Lowry, R.P., Baddoura, F.K., and Lakkis, F.G. 1996. Acute rejection of vascularized heart allografts in the absence of IFNgamma. Transplantation. 62:1908-1911.

37. Steiger, J., Nickerson, P.W., Steurer, W., Moscovitch-Lopatin, M., and Strom, T.B. 1995. IL-2 knockout recipient mice reject islet cell allografts. J. Immunol. 155:489-498.

38. Wekerle, T., et al. 2001. Peripheral deletion after bone marrow transplantation with costimulatory blockade has features of both activationinduced cell death and passive cell death. J. Immunol. 166:2311-2316.

39. Li, X.C., Wells, A.D., Strom, T.B., and Turka, L.A. 2000. The role of T cell apoptosis in transplantation tolerance. Curr. Opin. Immunol. 12:522-527.

40. Li, X.C., Strom, T.B., Turka, L.A., and Wells, A.D. 2001. T cell death and transplantation tolerance. Immunity. 14:407-416.

41. Issazadeh, S., et al. 2000. Role of passive T-cell death in chronic experimental autoimmune encephalomyelitis. J. Clin. Invest. 105:1109-1116.

42. Onodera, K., et al. 1998. Role of regulatory T cells in the "infectious" tolerance pathway in transplant recipients. Transplant Proc. 30:13-15.

43. Graca, L., Honey, K., Adams, E., Cobbold, S.P., and Waldmann, H. 2000. Cutting edge: anti-CD154 therapeutic antibodies induce infectious transplantation tolerance. J. Immunol. 165:4783-4786.

44. Hara, M., et al. 2001. Il-10 is required for regulatory T cells to mediate tolerance to alloantigens in vivo. J. Immunol. 166:3789-3796.

45. Waaga, A.M., et al. 2001. Regulatory functions of self-restricted MHC class II allopeptide-specific Th2 clones in vivo. J. Clin. Invest. 107:909-916.

46. Qin, S., et al. 1993. "Infectious" transplantation tolerance. Science. 259:974-976.

47. Wise, M.P., Bemelman, F., Cobbold, S.P., and Waldmann, H. 1998. Linked suppression of skin graft rejection can operate through indirect recognition. J. Immunol. 161:5813-5816.

48. Malvey, E.N., Jenkins, M.K., and Mueller, D.L. 1998. Peripheral immune tolerance blocks clonal expansion but fails to prevent the differentiation of Th1 cells. J. Immunol. 161:2168-2177.

49. Malvey, E.N., Telander, D.G., Vanasek, T.L., and Mueller, D.L. 1998. The role of clonal anergy in the avoidance of autoimmunity: inactivation of autocrine growth without loss of effector function. Immunol. Rev. 165:301-318.

50. Salama, A.D., Remuzzi, G., Harmon, W.E., and Sayegh, M.H. 2001 Challenges to achieving clinical transplantation tolerance. J. Clin. Invest. 108:943-948. doi:10.1172/JCI200114142. 